\title{
Cardiac reprogramming factor Gata4 reduces postinfarct cardiac fibrosis through direct repression of the profibrotic mediator snail
}

\author{
Megumi Mathison, MD, PhD, ${ }^{a}$ Vivek P. Singh, PhD, ${ }^{a}$ Deepthi Sanagasetti, MS, ${ }^{a}$ Lina Yang, PhD, \\ Jaya Pratap Pinnamaneni, MS, ${ }^{\mathrm{a}}$ Jianchang Yang, $\mathrm{MD}, \mathrm{PhD},{ }^{\mathrm{a}}$ and Todd K. Rosengart, $\mathrm{MD}^{\mathrm{a}, \mathrm{b}}$
}

\begin{abstract}
Objective: The administration of a variety of reprogramming factor cocktails has now been shown to reprogram cardiac fibroblasts into induced cardiomyocyte-like cells. However, reductions in ventricular fibrosis observed after reprogramming factor administration seem to far exceed the extent of induced cardiomyocyte-like cell generation in vivo. We investigated whether reprogramming factor administration might primarily play a role in activating antifibrotic molecular pathways.
\end{abstract}

Methods: Adult rat cardiac fibroblasts were infected with lentivirus encoding the transcription factors Gata4, Mef2c, or Tbx5, all 3 vectors, or a green fluorescent protein control vector. Gene and protein expression assays were performed to identify relevant antifibrotic targets of these factors. The antifibrotic effects of these factors were then investigated in a rat coronary ligation model.

Results: Gata4, Mef2c, or Tbx5 administration to rat cardiac fibroblasts in vitro significantly downregulated expression of Snail and the profibrotic factors connective tissue growth factor, collagen1a1, and fibronectin. Of these factors, Gata4 was shown to be the one responsible for the downregulation of the profibrotic factors and Snail (mRNA expression fold change relative to green fluorescent protein for Snail, Gata4: $0.5 \pm 0.3$, Mef2c: $1.3 \pm 1.0$, Tbx5: $0.9 \pm 0.5$, Gata4, Mef2c, or Tbx5: $0.6 \pm 0.2, P<.05)$. Chromatin immunoprecipitation quantitative polymerase chain reaction identified Gata4 binding sites in the Snail promoter. In a rat coronary ligation model, only Gata4 administration alone improved postinfarct ventricular function and reduced the extent of postinfarct fibrosis.

Conclusions: Gata4 administration reduces postinfarct ventricular fibrosis and improves ventricular function in a rat coronary ligation model, potentially as a result of Gata4-mediated downregulation of the profibrotic mediator Snail. (J Thorac Cardiovasc Surg 2017;154:1601-10)



Gata4 administration alone inhibited postinfarct ventricular fibrosis.

\section{Central Message}

We propose a direct molecular mechanism by which Gata4 decreases cardiac fibrosis by downregulating the expression of the profibrotic transcription factor Snail in cardiac fibroblasts.

\section{Perspective}

Gata4 administration alone could significantly reduce postinfarct fibrosis and improve cardiac function, although Gata4 administration alone is incapable of generating iCMs. The ability of Gata4 to thereby independently inhibit postinfarct fibrosis may be an important new means of treating heart failure separate from the recent focus on the role of Gata4 as a cellular reprogramming factor.

See Editorial Commentary page 1611.

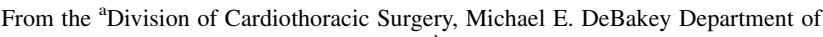
Surgery, Baylor College of Medicine; and ${ }^{\mathrm{b}}$ Department of Cardiovascular Surgery, Texas Heart Institute, Houston, Tex.

This study was funded by the National Heart Lung and Blood Institute (1R01HL121294-01A1 [to T.K.R.]) and supported in part by the Mouse Phenotyping Core at Baylor College of Medicine with funding from the National Institutes of Health (U54 HG006348).

Received for publication Dec 7, 2016; revisions received June 11, 2017; accepted for publication June 14, 2017; available ahead of print July 12, 2017.

Address for reprints: Todd K. Rosengart, MD, 1 Baylor Plaza, MS 390, Houston, TX 77030 (E-mail: todd.rosengart@bcm.edu).

$0022-5223 / \$ 36.00$

Copyright (c) 2017 by The American Association for Thoracic Surgery

http://dx.doi.org/10.1016/j.jtcvs.2017.06.035
}

The recent discovery that cardiac fibroblasts could be transdifferentiated into induced cardiomyocyte-like cells (iCMs) led to the reporting by our group and others that administration of a transcription factor "cocktail" (eg, Gata4, Mef2c, and Tbx5 [GMT]) into areas of

U. Scanning this QR code will
take you to a supplemental
$\square$ video and figures for the
article.




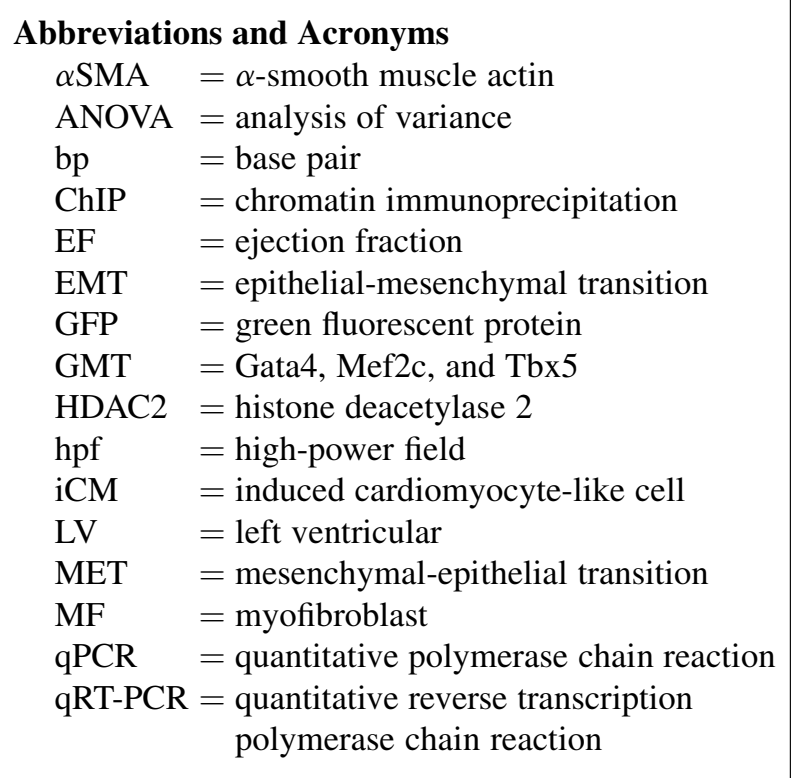

myocardial scar resulted in the apparent replacement of large areas of postinfarct scar with functional myocardium and up to a $37 \%$ accompanying increase in ventricular function. ${ }^{1-5}$ We and others ${ }^{1-4}$ also observed that reductions in infarct size appeared to far exceed the extent of scar repopulation with iCMs, suggesting that yet unknown antifibrotic mechanisms may underlie reprogramming efficacy.

Consistent with these observations, we have also noted that GMT administration significantly reduced the ventricular infarct population of myofibroblasts (MFs), which are thought to be differentiated from quiescent cardiac fibroblasts by inflammatory stimuli such as transforming growth factor- $\beta$ and responsible for inducing tissue fibrosis. ${ }^{6}$ In normal circumstances, MFs are dissipated through apoptosis after healing is complete, limiting the extent of fibrotic reaction to inflammatory stimuli. In pathologic states, MF activation persists and leads to excessive degrees of fibrosis, but the specific molecular mechanisms underlying this prolonged activation remain unclear.

Given these observations, we hypothesized that 1 or several of the transcription factors that have been used to induce cardiac cellular reprogramming might inhibit MF differentiation as an antifibrotic mechanism distinct from iCM generation. We accordingly sought to investigate the potential role of the cardiac reprogramming factors GMT in suppressing profibrotic processes in vitro and in vivo.

\section{MATERIALS AND METHODS \\ Vectors and Cells}

Lentivirus vectors encoding GMT and the marker gene green fluorescent protein (GFP) and a control lentivirus construct encoding
GFP alone were prepared as previously described. ${ }^{1}$ Adult cardiac fibroblasts were harvested from 8- to 10-week-old male Sprague Dawley rats (Harlan, Indianapolis, Ind) using standard cell isolation protocols that were approved by the Baylor College of Medicine Institutional Animal Care and Use Committee. ${ }^{7}$ All animals received humane care in compliance with the Guide for the Care and Use of Laboratory Animals.

Harvested cells were maintained in Iscove's Modified Dulbecco's Medium culture media (Life Technologies, Carlsbad, Calif) supplemented with $10 \%$ fetal bovine serum (Gibco, Gaithersburg, Md) at a concentration of $1 \times 10^{5}$ cells $/ \mathrm{mL}$. The cells were then seeded onto a $10-\mathrm{cm}$ dish with a total of $1 \times 10^{6}$ cells for chromatin immunoprecipitation (ChIP) quantitative polymerase chain reaction (qPCR) and a 6-well dish with a total of $10^{5}$ cells/well for Western blot and quantitative real-time polymerase chain reaction (qRT-PCR). Second passage cells were then infected with $1 \times 10^{5}$ TU of lentivirus encoding GMT, a combination of these vectors $\left(1 \times 10^{5} \mathrm{TU}\right.$ dose of each vector; GMT); or an equivalent dose of GFP (negative control). ${ }^{1}$ Cells were harvested for ChIP qPCR, analysis 5 days after the infection, and qRT-PCR and Western analyses 14 days after the infection.

\section{Quantitative Polymerase Chain Reaction Real-Time Polymerase Chain Reaction}

To perform qRT-PCR, total RNA was isolated from cultured cells or primary tissues using Trizol, and cDNA was generated from $1 \mu \mathrm{g}$ RNA using the Superscript III first strand synthesis kit (Invitrogen, Carlsbad, Calif). qRT-PCR was then performed using SYBR Green Master mix (Life Technologies) on a ViiA7 (Life Technologies). Results were normalized by comparison with glyceraldehyde 3-phosphate dehydrogenase. Primer sequences are listed in Table 1.

\section{Chromatin Immunoprecipitation-Quantitative Polymerase Chain Reaction}

Cells designated for ChIP analysis were first cross-linked with formaldehyde, lysed, and sonicated, using ChromaFlash Chromatin Extraction kit (p-2001, Epigenetek, Farmingdale, NY). ChIPs were conducted with a polyclonal anti-Gata4 (sc-25310, Santa Cruz, Dallas, Tex), anti-HDAC2 (ab12169, abcam, Cambridge, Mass), or anti-histone 3 lysine 27 acetylation (4729, abcam) antibody.

To find the theoretic Gata4 binding sites, we analyzed the 6000 base pairs (bp) located before transcription starting point of the Snail gene using the following sequences that were previously reported bound by Gata4: TGATAA, AGATAA, AGATAG and TGATAG. ${ }^{8}$ In addition, an extra potential binding sequence, AGATAT was analyzed with the Pattern Search for Transcription Factor Binding Sites program (http://www.generegulation.com/cgi-bin/pub/programs/patch/bin/patch.cgi). A region (Region I) that contains none of these sequences was included in this analysis (Figure E1).

For ChIP-qPCR assays, all primers were synthesized by Sigma (St Louis, Mo). These sequences are listed in Table 1. Fold enrichment of the PCR product was calculated after normalization with input (no antibody added) of all 3 types of infected cells.

\section{Rat Coronary Artery Ligation Myocardial Infarction Model}

After approval of the protocol by the Baylor College of Medicine Institutional Animal Care and Use Committee, myocardial infarction was created via proximal left anterior descending coronary artery ligation in adult male Sprague Dawley rats $\left(\mathrm{n}=9\right.$ /group). ${ }^{1,2}$ Immediately after coronary ligation, animals received direct administration (total of $100 \mu \mathrm{L}, 5$ uniformly distributed $20 \mu \mathrm{L}$ injections) into the myocardial infarct zone of lentivirus $\left(1 \times 10^{5} \mathrm{TU}\right)$ encoding Gata4, GFP, or a combination of GMT $\left(3 \times 10^{5}\right.$ total dose GMT $){ }^{1,2}$ Four animals that did not survive to study completion were excluded from the final analysis 
TABLE 1. Primer sequences used for quantitative reverse transcriptase polymerase chain reaction and chromatin immunoprecipitation quantitative polymerase chain reaction assays

\begin{tabular}{llll}
\hline Gene (rat) & GeneBank accession & Forward primer $\left(\mathbf{5}^{\prime} \mathbf{-} \mathbf{3}^{\prime}\right)$ & Reverse primer $\left(\mathbf{5}^{\prime} \mathbf{- \mathbf { 3 } ^ { \prime } )}\right.$ \\
\hline GAPDH & NM_017008.3 & ggcacagtcaaggctgagaatg & atggtggtgaagacgccagta \\
Snail & NM_053805.1 & gaagatgcacatccgaagc & agtgggagcaggagaagg \\
CTGF & NM_022266.2 & gctgacctagaggaaaacattaaga & ccggtaggtcttcacactgg \\
Collagen1a1 & NM_0533304.1 & catgttcagctttgtggacct & gcagctgacttcagggatgt \\
Fibronectin & NM_019143.2 & cagcccetgattggagtc & tgggtgacacctgagtgaac \\
E-Cadherin & NM_031334.1 & gatcctggccctcetgat & tctttgaccaccgttctcct \\
N-Cadherin & NM_031333.1 & ccatcatcgcgatacttctg & ccataccacgaacatgagga \\
Region I & N/A & gcagtcagtgctcttaagcc & gtcaccaagaatgcccacctcc \\
Region II & N/A & ggcagtagatcccccagaaccag & gggtcttgcactttctaggc \\
Region III & N/A & tacagggcaaggcagaggaca & ccagagaccacctgaaggaaagg \\
Region IV & N/A & gcttcctgtctctgcctcc & ggtgtgtgccaccattgcc \\
Region V & N/A & gtcagggctgtcgtgttgtttg & gctttcctcgagagagtgtctg \\
\hline
\end{tabular}

GADPH, Glyceraldehyde 3-phosphate dehydrogenase; $C T G F$, connective tissue growth factor; $N / A$, not available.

( 1 animal received Gata4, and 3 animals received GMT). All 6 animals with ejection fractions (EFs) at baseline (3 days after coronary ligation) that were outside the $75 \%$ boundary (ie, median \pm quartile boundary, $49 \pm 14$ (Figure E2) were excluded from the final analysis, on the basis of Tukey's outlier detection method ( 1 received Gata4 and 5 received GFP). Therefore, the final number for the outcome studies was Gata $4=7$, GMT $=6$, and GFP $=4$.

\section{Echocardiography}

Echocardiography was performed using 3\% isoflurane using a Veno 770 Imaging System (VisualSonics, Toronto, Ontario, Canada). ${ }^{1,2}$ Parasternal long-axis and short-axis images were obtained by investigators who were unaware of the treatment groups. The left ventricular (LV) end-systolic and end-diastolic diameters and anterior and posterior wall thicknesses were measured from M-mode tracings acquired at the level of the papillary muscle. The change in EF from baseline was calculated as [(EF at day 28 after the surgery) - (EF at day 3$)] /(E F$ at day 3$)$. The LV wall thickening was calculated as [(wall thickness at day 28 after surgery) - (wall thickness at day 3)]/(wall thickness at day 3 ).

\section{Histologic Examination}

Cardiac specimens were harvested 4 weeks after coronary ligation and vector administration. ${ }^{1,2}$ Briefly, animals were exsanguinated under deep anesthesia by an incision made in the right atrium, after which the beating hearts were perfused with normal saline and fixed with phosphate-buffered saline ( $\mathrm{pH} 7.2)$ containing $4 \%(\mathrm{wt} / \mathrm{vol})$ paraformaldehyde. The hearts were then harvested and rinsed with saline.

Excised hearts were fixed with $4 \%$ paraformaldehyde for 24 hours and then with $2 \%$ paraformaldehyde for 48 hours at $4^{\circ} \mathrm{C}$. Each heart was cut transversally, and 2 slices $(2-3 \mathrm{~mm})$ were obtained, each immediately above or below the transverse centerline of the infarct region, which was readily identifiable by gross inspection. ${ }^{1,2}$ After paraffin embedding of these slices, two $5-\mu \mathrm{m}$-thick sections were obtained at $115-\mu \mathrm{m}$ intervals.

Seven sections per animal were stained with Masson-Trichrome. The fibrotic area (blue) and the nonfibrotic region (red) were outlined using Adobe Photoshop CS5 software (Adobe, San Jose, Calif) and quantified with MATLAB and Simulink software (MathWorks, Natick, Mass). The percent of the LV wall area with fibrosis was calculated as follows: (total blue pixels from all sections/total blue plus red pixels from all sections) $\times 100$.

For identification of MFs and cells expressing Snail, we analyzed the 2 sections per animal demonstrating the greatest cross-sectional area of fibrosis, as determined by trichrome staining. ${ }^{1,2}$ These sections were stained for $\alpha$-smooth muscle actin ( $\alpha$ SMA; ab5694, abcam) and Snail (ab180714, abcam). Labeled cells were counted at $200 \times$ magnification $\alpha \mathrm{SMA}^{+}$cells found in the vasculature and endocardium were not counted as MFs because these structures contain $\alpha$ SMA-positive cells. ${ }^{1,2}$ Because Gata4 has been reported to be an angiogenic factor, we also counted $\alpha$ SMA positive vessels in 4 representative fields ( 2 each from the right or left peri-infarct region). ${ }^{9,10}$

Cardiomyocyte diameter was measured at $400 \times$ magnification of cardiomyocytes found in the peri-infarct (anterior, lateral) regions subtended by the ligated left anterior descending coronary artery and the noninfarcted (posterior) LV regions. The slide demonstrating the greatest area of fibrosis, as identified by Masson's Trichrome staining (Figure E3, $A$ and $B$ ), was selected for each animals, because this section was expected to most likely include the regions into which vector was injected (ie, as noted earlier, injections were made into the infarct zone). In each slide, 100 longitudinally oriented cardiomyocytes from each 3 regions, anterior, lateral, and posterior, were examined, and the diameters were defined. The mean value of 100 measurements represented 1 sample from each position in each animal.

\section{Statistical Analysis}

Statistical analysis was performed using SAS version 9.2 (SAS Institute Inc, Cary, NC). Data are presented as the mean \pm standard deviation, unless otherwise indicated. The normality of the data was first examined using a Kolmogorov-Smirnov test. If the data have normal distribution, the analysis of variance (ANOVA) test was used. If the data did not meet normality assumption, a Kruskal-Wallis test was used. If ANOVA or Kruskal-Wallis test was significant for more than 2-group comparison, Bonferroni correction for ANOVA or Wilcoxon rank test was followed for each pair comparison.

\section{RESULTS}

Administration of GMT or Gata4 alone significantly downregulates Snail and other profibrotic genes. Rat cardiac fibroblasts treated with a cocktail of lentivirus vectors encoding GMT for 14 days demonstrated $50 \% \pm 6 \%$ downregulation $(P<.001)$ of the expression of the profibrotic transcription factor Snail at both the transcriptional and translational levels (Figure 1). Because 


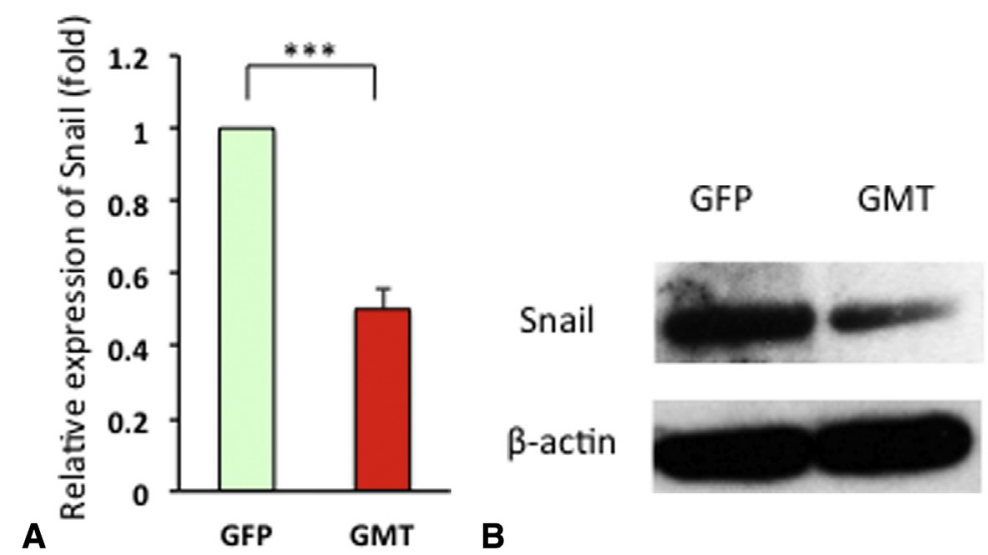

FIGURE 1. Expression of Snail in cardiac fibroblasts infected with lentivirus encoding GMT or a GFP control vector. A, qPCR analysis of Snail mRNA expression 14 days after treatment of rat cardiac fibroblasts with GMT or a GFP control vector, as described in the "Materials and Methods" section $(\mathrm{n}=3$ biological replicates, each performed in triplicate). Data are shown as fold change relative to GFP. $* * * P<.0001$. B, Immunoblot representation of Snail protein expression after treatment of cells with lentivirus expressing GFP (negative control) or a combination of GMT, as described $(\mathrm{n}=3$, representative figure is shown). GFP, Green fluorescent protein; GMT, Gata4, Mef2c, and Tbx5.

it has been shown that Snail is an important profibrotic master transcription factor, we tested whether Snail is involved in fibrosis suppression by Gata4. ${ }^{11-13}$ When rat cardiac fibroblasts were infected with lentivirus expressing GMT alone for 14 days, only Gata4 induced downregulation of Snail expression (Snail mRNA expression fold change relative to GFP for Gata4: $0.5 \pm 0.3$, Mef2c: $1.3 \pm 1.0$, Tbx5: $0.9 \pm 0.5$, and GMT: $0.6 \pm 0.2 ; P<.05$ for Gata4 vs GFP and for GMT vs GFP) (Figure 2, $A$ ). Furthermore, only Gata4 also downregulated the expression of the profibrotic genes connective tissue growth factor, collagen 1a1, and fibronectin (Figure 2, B). Analogous data after GMT administration were obtained by Western analysis (Figure 2, C).

\section{Gata4 Directly Binds to the Snail Promoter}

We next sought to determine whether Gata4 might downregulate Snail expression by binding to the Snail promoter. ChIP-qPCR analysis demonstrated that Gata4 binds to the specific promoter regions of Snail $(-3483 \mathrm{bp}$ $\sim-3297$ bp, Region III, and -1798 bp $\sim-1597$ bp, Region V) (Figure 3).

We next hypothesized that Gata4 suppresses Snail by deacetylation of the Snail promoter in cooperation with histone deacetylase 2 (HDAC2). ${ }^{14}$ We accordingly observed that HDAC2 occupied the same Snail promoter regions as the region III and V Gata4 binding sites noted earlier $(P<.01)$, and that there was reduced enrichment of histone 3 lysine 27 acetylation at region III $(P<.01)$.


FIGURE 2. Expression of Snail and profibrotic factors connective tissue growth factor, collagen1a1, and fibronectin in cardiac fibroblasts infected with GMT. A, qPCR analysis of mRNA expression of Snail expression 14 days after treatment of rat cardiac fibroblasts with various reprogramming factors, as described in "Materials and Methods" section. B, qPCR analysis of mRNA expression of the profibrotic mediators connective tissue growth factor, collagen1a1, and fibronectin $(\mathrm{n}=3$ biological replicates, each performed in triplicate). Data are shown as fold change relative to GFP. $* P<.05$, ${ }^{*} * P<.01$ compared with GFP. C, Representative Western blot images for Snail, connective tissue growth factor, collagen1a1, fibronectin, and $\beta$-actin 14 days after treatment of rat cardiac fibroblasts with Gata4 alone, GMT, a GFP control vector, or without treatment $(\mathrm{NT})(\mathrm{n}=3$, representative figure is shown). GFP, Green fluorescent protein; GMT, Gata4, Mef2c, Tbx5; $C T G F$, connective tissue growth factor; $N T$, no treatment. 


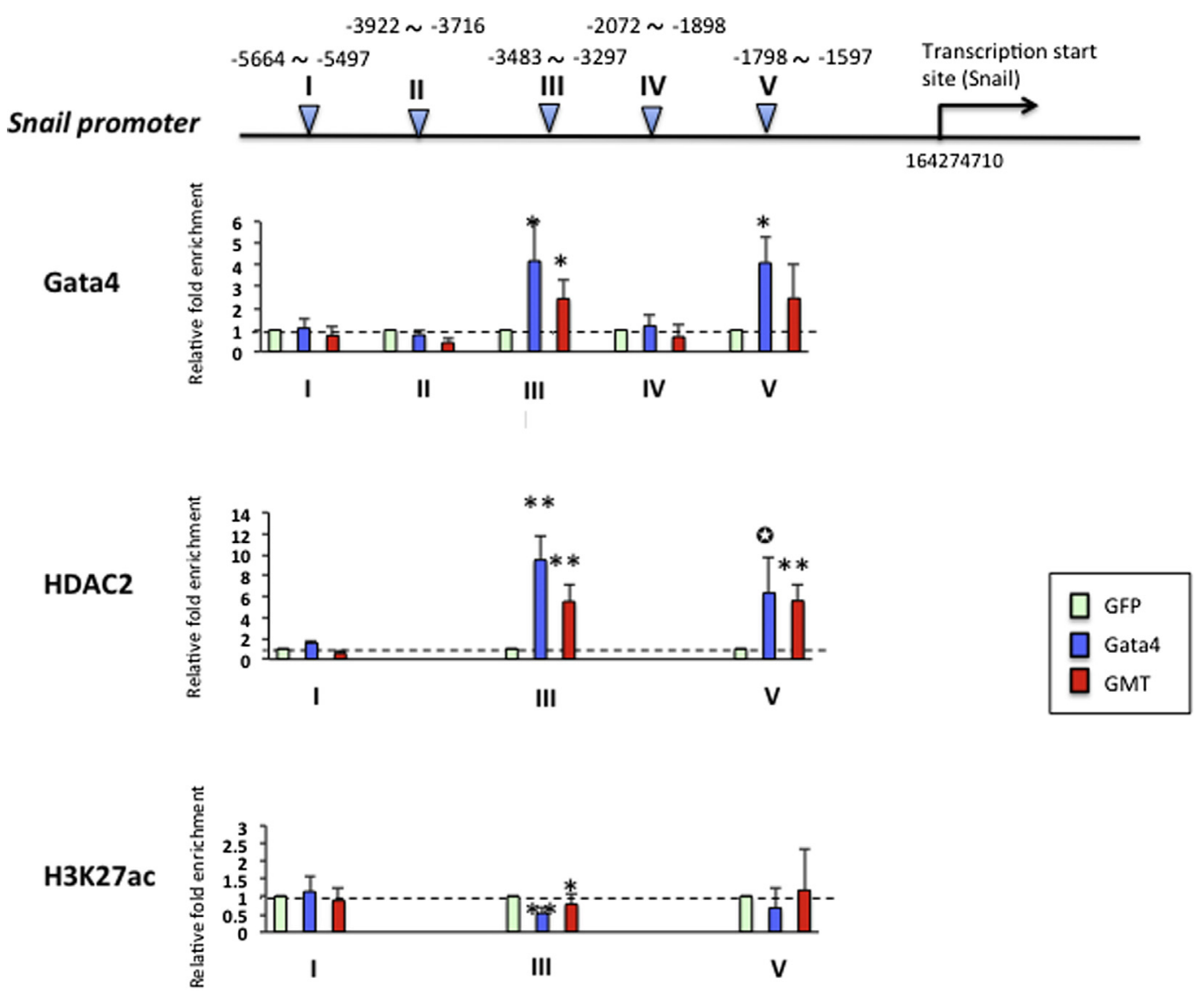

FIGURE 3. Gata4 co-occupies the same promoter regions of Snail as HDAC2, and histone 3 lysine 27 acetylation is decreased at these regions. ChIP qPCR was performed as described in the "Materials and Methods" section. Data indicate that regions III and V were bound by Gata4, and Gata4 and HDAC2 co-occupied these regions. Histone 3 lysine 27 acetylation was decreased in region III. Graphs represent fold enrichment compared with GFP group after normalization with input. $\mathrm{n}=3$ per group. ${ }^{*} P<.05$. $* *<.01 . \otimes P=.05 . G F P$, Green fluorescent protein; GMT, Gata4, Mef2c, Tbx5.

\section{Gata4 Shifts the Expression Ratio of Epithelial and Mesenchymal Cellular Markers}

Because Snail has been shown to stimulate profibrotic epithelial-mesenchymal cellular transition and endothelialmesenchymal transition, and suppression of Snail has been shown to induce antifibrotic mesenchymal-epithelial transition (MET), ${ }^{11-13}$ we hypothesized that Gata4 might inhibit fibrosis by rebalanced MET over epithelialmesenchymal transition (EMT). We accordingly found that Gata4 or GMT administration to rat cardiac fibroblasts in vitro increased expression of the epithelial cell marker E-cadherin compared with expression of the mesenchymal cell marker N-cadherin. The increase of E-cadherin/ $\mathrm{N}$-cadherin ratio indicates MET transition to a nonfibrogenic cell phenotype (Figure 4).

\section{Gata4 Administration Decreases Postinfarct Cardiac Fibrosis In Vivo}

Administration of lentivirus encoding Gata4 alone was associated with significantly less ventricular fibrosis 4 weeks after rat coronary artery ligation compared with animals treated with a GFP control vector (percent of LV myocardial wall with fibrosis: Gata4: $9 \% \pm 4 \%$, GMT: $15 \% \pm 9 \%$, and GFP: $20 \% \pm 8 \% ; P<.05$ for Gata4 vs GFP) (Figure 5).

MF ( $\alpha$ SMA positive) cell number was likewise significantly decreased in Gata4 versus GMT or GFP-treated animals $(128$ cells/high-power field [hpf] \pm 68 cells/hpf, 323 cells/hpf \pm 106 cells/hpf, and 489 cells/hpf \pm 303 cells/hpf, respectively; $P<.01$ for Gata4 vs GMT, $P<.05$ Gata4 vs GFP) (Figure 6, A). Snail positive cells were also reduced in Gata4- and GMT-treated animals versus GFP-injected animals, which demonstrated abundant Snail positive cells in infarct zones (51 cells/ hpf \pm 55 cells/hpf, 70 cells/hpf \pm 64 cells/hpf, and 167 cells/hpf \pm 40 cells/hpf, respectively; $P<.05$ for Gata4 vs GFP, $P=.08$ for GMT vs GFP) (Figure $6, B$ ).

\section{Gata4 Delivery Improves Postinfarct Cardiac Function In Vivo}

GMT administration and Gata4 administration alone resulted in significant improvements in postinfarct EF from baseline 3 days after coronary ligation and reprogramming factor administration to final analysis 4 week later (GMT: $51 \% \pm 3 \%$ to $59 \% \pm 6 \% ; P<.05$ ), Gata4: 


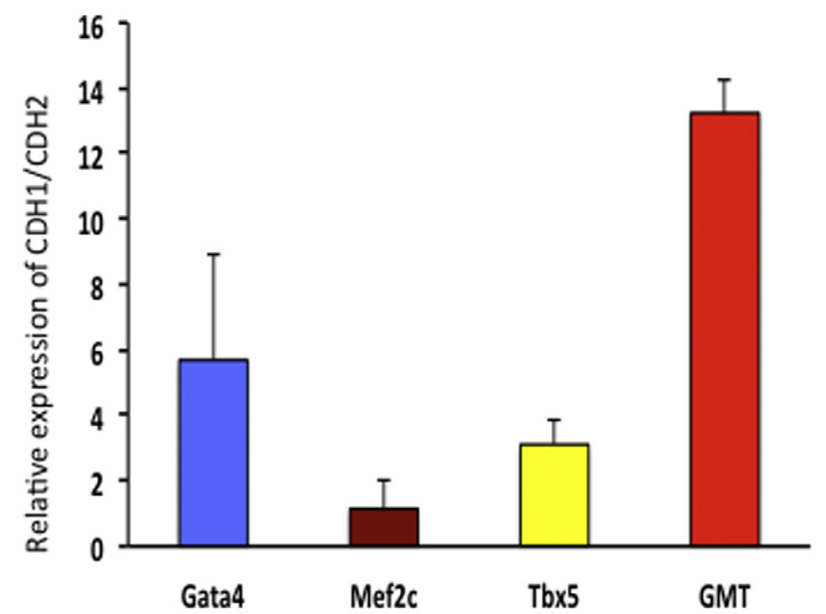

FIGURE 4. The ratio of E-cadherin/N-cadherin is increased in Gata4- and GMT-infected cells. qPCR analysis of E-cadherin and N-cadherin mRNA expression 14 days after treatment of rat cardiac fibroblasts with GMT, or GFP control vector, as described in the "Materials and Methods" section ( $n=3$ biological replicates, each performed in triplicate). Representation of the ratio of E-cadherin/ $\mathrm{N}$-cadherin for each group, using the values as fold change relative to GFP. $P=.16$ ANOVA test for 5 groups together. GMT, Gata4, Mef2c, Tbx5.

$46 \% \pm 4 \%$ to $56 \% \pm 6 \% ; P<.01$ ) (Figure 7 ). No improvement in EF was observed in GFP treated animals $(48 \% \pm 4 \%$ to $45 \% \pm 5 \%)$.

The average change in EF for each animal from baseline to 4-week follow-up was significantly greater in the Gata4and GMT-treated groups versus the GFP group $(22 \% \pm 14 \%, 15 \% \pm 7 \%$, and $-6 \% \pm 3 \%$, respectively; $P<.001$ for Gata4 vs GFP and $P<.01$ for GMT vs GFP).

\section{Gata4 Effects on Cardiomyocyte Hypertrophy}

We likewise noted that diameters of cardiomyocytes found in the posterior ventricular wall were significantly greater in hearts treated with Gata4 or GMT compared with controls $(P<.001$ for Gata4 vs GFP, $P<.01$ for GMT vs GFP) and were significantly greater in the lateral wall of the Gata4-treated group compared with the GFP control group $(P<.01$ for Gata4 vs GFP, $P<.05$ for GMT vs GFP) (Figure E3, $C$ ).

A corresponding trend toward increased LV free wall thickening, as measured by M-mode echocardiographic analysis of the posterior LV wall, was also observed after Gata4 administration compared with GFP controls (posterior wall thickening: Gata4: 46\% \pm 40\%, GMT: $16 \% \pm 28 \%$, and GFP: $-1 \% \pm 22 \%$; ANOVA: $P=.08)$. Intragroup variability precluded meaningful results of anterior wall thickening analysis, likely because of the effects of infarction on this metric (Table 2).

\section{DISCUSSION}

The administration of various cellular reprogramming factor combinations into myocardial tissues has now



FIGURE 5. Extent of LV wall fibrosis is decreased after Gata4 administration. The percent of LV myocardial wall area demonstrating fibrosis, as determined by trichrome staining of sections of myocardial tissue harvested 4 weeks after coronary ligation and administration of lentivirus encoding Gata4, GMT, or a GFP control vector, as described in the "Materials and Methods" section (Gata4: $\mathrm{n}=7$, GMT: $\mathrm{n}=6$, GFP: $\mathrm{n}=4) . * P<.05$. GMT, Gata4, Mef2c, and Tbx5; GFP, green fluorescent protein.

repeatedly been shown to lead to improvements in postinfarct ventricular function. ${ }^{1-5}$ This has been presumed to be due to the ability of these reprogramming factors to transdifferentiate resident cardiac fibroblasts into iCMs. Relatively ignored, however, has been the observation that reprogramming factor administration significantly reduces postinfarction fibrosis. ${ }^{1-5}$

We offer evidence that the unanticipated reductions in postinfarct fibrosis observed after reprogramming factor administration can be attributed to primary antifibrotic effects of Gata4 alone. This novel observation can be traced to the origins of the use of GMT as a reprograming cocktail, formulated by Ieda and colleagues by serial deletions from larger reprograming cocktails. ${ }^{15}$ Because subsequent studies were predominantly based on Srivastava and colleagues' GMT formulation, the effects of individual reprogramming factors (ie, GMT) on fibrosis have not, to our knowledge, been explored.

We now demonstrate that the antifibrotic effects of Gata4 appear to occur via Gata4-mediated downregulation of the profibrotic zinc finger transcription factor Snail and its downstream effectors. Further, we have now been able to show that Gata4-mediated Snail downregulation is also associated with the reduction in the number and activity of profibrotic $\mathrm{MFs}^{16-18}$ and the rebalancing of the 



QSMA
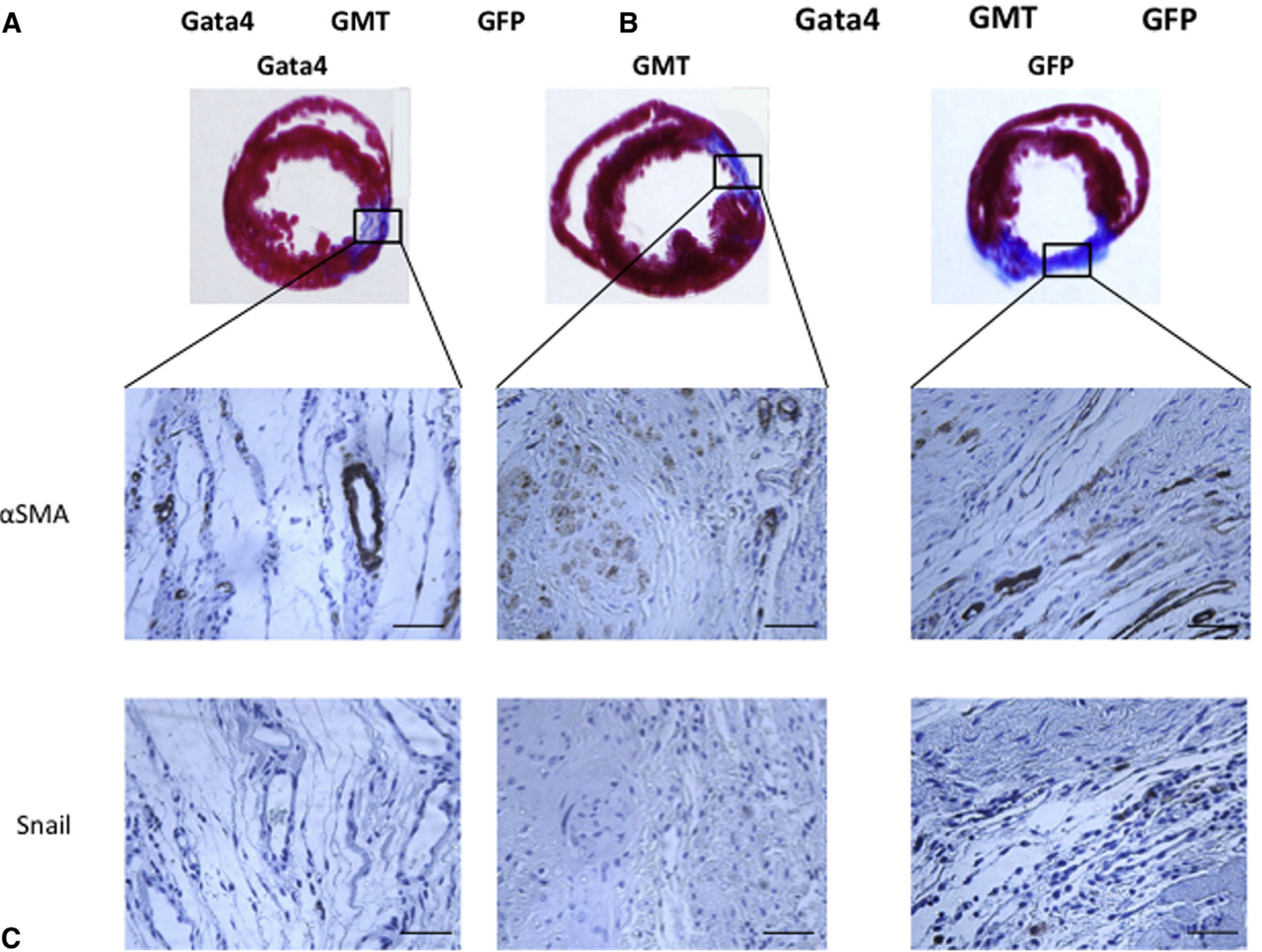

FIGURE 6. GMT and Gata4 administration decrease the number of cells expressing the MF marker $\alpha$ SMA and the profibrotic transcription factor Snail. A, Representation of the number of cells per hpf expressing the MF marker $\alpha$ SMA in sections of myocardial tissue harvested 4 weeks after coronary ligation and administration of lentivirus encoding Gata4, GMT or a GFP control vector, as described in the "Materials and Methods" section (Gata4: $\mathrm{n}=7$, GMT: $\mathrm{n}=6$, GFP: $\mathrm{n}=4) . * P<.05, * * P<.01$ compared with GFP. B, Representation of the number of cells expressing Snail in infarct regions, quantified as described in the "Materials and Methods" section. $* P<.05$. C, Photomicrographs of representative sections of infarct zones (fibrosis depicted by blue trichrome staining), demonstrating extent of staining for $\alpha$ SMA (top) or Snail (bottom), obtained as described in the "Materials and Methods" section. Bars represent $100 \mu \mathrm{m} . \alpha S M A, \alpha$-smooth muscle actin; GMT, Gata4, Mef2c, and Tbx5; GFP, green fluorescent protein. 


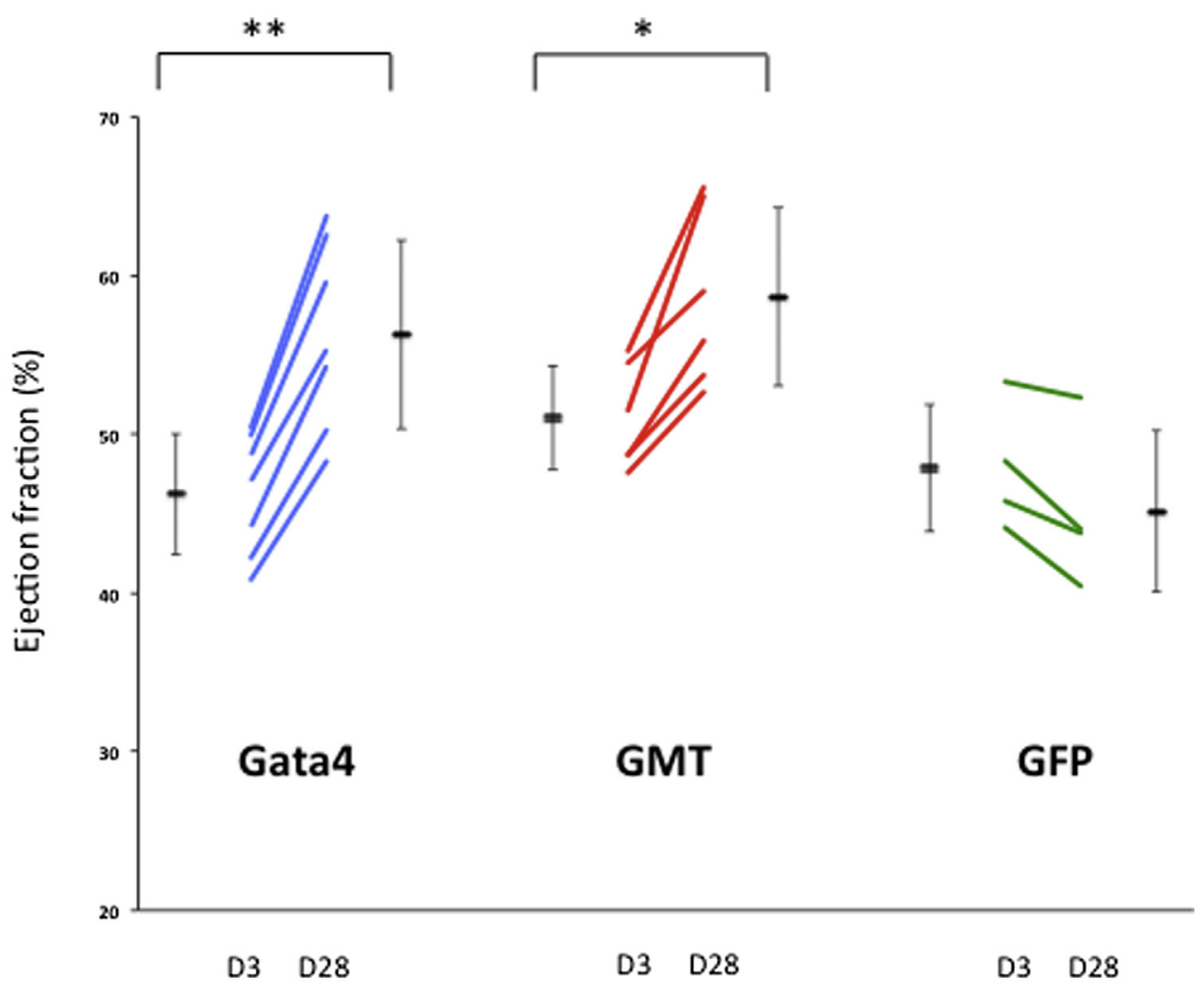

FIGURE 7. Echocardiographic analysis of global ventricular function after administration of Gata4, GMT, or GFP control vectors. Global EF at specified time points for each animal obtained from echocardiographic studies performed, as described in the "Materials and Methods" section, 3 days after coronary ligation and administration of Gata4 or GMT or GFP (D3) and 4 weeks later (D28). Gata4: $\mathrm{n}=7$, GMT: $\mathrm{n}=6$, GFP: $\mathrm{n}=4$. Mean and standard deviation for EF 3 days after the surgery are depicted at left, and 4 weeks after the surgery, at right. $* P<.05$. $* * P<.01$. GMT, Gata4, Mef2c, and Tbx5; GFP, green fluorescent protein.

profibrotic cellular differentiation pattern called "EMT" to an antifibrotic pattern termed "MET."11-13 Consistent with our findings, Song and colleagues ${ }^{19}$ previously observed decreased liver fibrosis after incorporating Gata4 in their hepatic reprogramming cocktail. In addition, observations that cardiomyocyte-specific Gata4 deletion did not increase fibrosis (whereas nonspecific Gata4 deletion did) further support the fibroblast/MF (EMT/MET) axis as central to the antifibrotic effects of Gata4 (Figure E4). ${ }^{20}$

Although Gata4 possesses complex gene activator and suppressor roles, depending on its specific epigenetic gene regulatory interactions, we now demonstrate that Gata4 acts in synergy with the epigenetic gene regulator HDAC2 to repress Snail expression through HDAC2-Gata4 complex. $^{21-25}$ Given the promiscuous effects and therefore complex challenges to the therapeutic manipulation of HDAC, the relative specificity of Gata4 may represent a compelling postinfarct therapeutic strategy.

Beyond these antifibrotic effects of Gata4, we are also intrigued by our observation that Gata4 alone improves postinfarct ventricular function equivalently to "complete" GMT transdifferentiating cocktails, especially because Gata4 alone is incapable of generating iCMs. ${ }^{415}$ Given that essentially all reprogramming studies to date have incorporated Gata4 administration or a combination of factors know to induce Gata4, ${ }^{1-5,26}$ the considerations suggest that improvements in postinfarct ventricular function now commonly ascribed to $\mathrm{ICM}$ generation instead may be largely due to the Gata4 effects we now report. In this regard, our use of a lentivirus

TABLE 2. Left ventricular wall thickness

\begin{tabular}{|c|c|c|c|c|c|c|}
\hline & \multicolumn{3}{|c|}{ End-systolic anterior wall thickness } & \multicolumn{3}{|c|}{ End-systolic posterior wall thickness } \\
\hline & Day 3 (mm) & Day $28(\mathbf{m m})$ & $\%$ change & Day 3 (mm) & Day $28(\mathbf{m m})$ & $\%$ change \\
\hline Gata4 & $1.8 \pm 0.3$ & $2.2 \pm 0.2$ & $79 \pm 53$ & $1.7 \pm 0.2$ & $2.3 \pm 0.2$ & $46 \pm 15$ \\
\hline GMT & $2.1 \pm 0.2$ & $2.3 \pm 0.2$ & $15 \pm 22$ & $2.3 \pm 0.1$ & $2.6 \pm 0.2$ & $16 \pm 12$ \\
\hline GFP & $1.3 \pm 0.1$ & $1.8 \pm 0.6$ & $32 \pm 25$ & $2.3 \pm 0.1$ & $2.2 \pm 0.2$ & $-1 \pm 11$ \\
\hline
\end{tabular}

GMT, Gata4, Mef2c, and Tbx5; GFP, green fluorescent protein. 


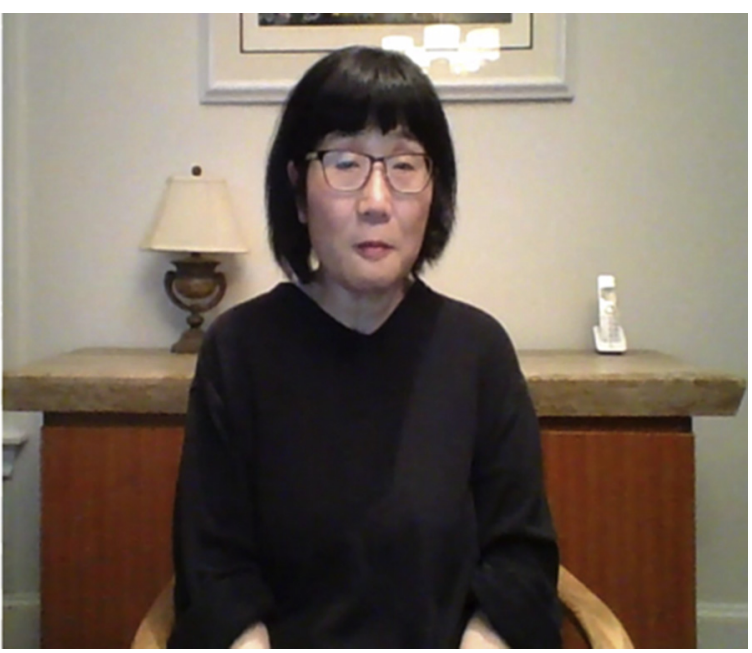

VIDEO 1. Summary of this study by Megumi Mathison. Video available at: http://www.jtcvsonline.org/article/S0022-5223(17)31354-5/fulltext.

vector, which infects both nondividing and dividing cells (ie, cardiomyocytes and fibroblasts), supports the premise of a direct Gata4 effect on cardiomyocyte structure (ie, as we observed in increased cardiomyocyte diameter or hypertrophy) and (potentially consequent) wall thickening.

Gata4 has been reported to be an angiogenic factor, ${ }^{9,10}$ and our analysis of $\alpha$ SMA positive vessels in our Gata4- and GMT-treated animals trended toward greater density (vessels/microscopic field) in these animals compared with GFP controls (data not shown). Substantiation of this observation would require more extensive studies of this outcome, including additional endothelial cell marker staining.

Additional studies are needed to further explore the new potential Gata4 mechanisms of the actions outlined in this report, including the possibility that hypertrophy effects could be deleterious to contractile function over time. Such effects have been shown after Gata4 administration, and evidence of increased fibrosis likewise has been shown with heterozygous deletion of this gene. ${ }^{9,20,27,28}$ The possibility of a primary antifibrotic effects of Gata4, as suggested by the current data, has not to our knowledge been previously considered.

\section{Study Limitations}

Despite the positive outcomes of the present report, the extremely complex nature of fibrotic/antifibrotic molecular pathways somewhat temper our enthusiasm for the utility of Gata4 as an antifibrotic agent, despite our and others' observations. ${ }^{29,30}$ For example, other transcription factors such as Slug, zinc-finger E-box-binding, and TWIST may play important roles in postinfarct myocardial fibrosis. We plan to use RNA-seq and ChIP-seq in future analyses to explore these questions.

Our qPCR analyses of the expression of Snail and other profibrotic factors by cardiac fibroblasts showed that Mef2c- or Tbx5-infected groups exhibited greater variation in expression levels compared with Gata4 or GMT infected groups (Figure 2). Although we were able to demonstrate differences between groups despite this variability, we do not have a good explanation for this variability between groups. In this context, we would note that we assayed RNA expression at 14 days after reprogramming factor infection based on original research in this field suggesting that cellular reprogramming occurs over several weeks, ${ }^{31,32}$ but additional studies demonstrated similar expression patterns as early as 5 days postinfection (data not shown).

Our observations of greater effects of Gata4 versus GMT on fibrosis and ventricular function further suggest complex and yet unexplained interplays between these agents. Potentially, these observations relate to the interplay of gene transfer vector infections, as we have previously considered. ${ }^{2}$ Our small animal model likewise limited our ability to more accurately track the effects of vector administration to the LV free wall, and remote effects of reprogramming factor administration beyond the region of vector administration cannot be ruled out in this model, as was observed in cardiomyocyte hypertrophy/ventricular thickening analyses. Larger animal studies would be needed to better resolve these effects.

Some of our "negative" outcomes (eg, GMT effect on fibrosis) may have been subject to a type II statistical error because our animal mortality rate and variability in myocardial infarction size in our coronary ligation model were somewhat larger than anticipated. On the other hand, compared with consistent baseline EFs of approximately $80 \%$ that we and others have observed for this model, ${ }^{1,2}$ the approximately $40 \%$ decrease in postligation EF readily allowed resolutions between groups for key outcomes of interest, including Gata4 effects on myocardial fibrosis and function.

On the other hand, our analysis of LV free wall thickening may have been impaired by variability between animals affected by differences in extensiveness of infarction zones. Despite this variability, we were able to observe trends toward differences between groups in posterior wall thickening, which would have been most remotely located away from infarct zones.

Finally, although our prior studies used delayed reprogramming factor administration 3 weeks after coronary ligation as a model of chronic, stable infarction, we used an immediate postligation time point for the current studies to maximize the opportunity for Gata4 to potentially counteract profibrotic mediators such as transforming growth factor, which are maximally expressed immediately after infarction. We are now planning studies examining delayed Gata4 administration.

\section{CONCLUSIONS}

Our current observations of the primary effects of Gata4 administration alone inhibiting postinfarct ventricular 
fibrosis and improving postinfarct ventricular function would help explain the disproportionately greater improvements in these indices observed after administration of reprogramming factor cocktails incorporating Gata4 (eg, "GMT") compared with the relatively inefficient (ie, $10 \%-15 \%$ ) induction of iCMs observed after reprogramming cocktail administration in vivo (Video 1$){ }^{3}$ We currently provide evidence that such Gata4-mediated effects occur through its downregulation of the expression of the profibrotic transcription factor Snail in cardiac fibroblasts. Although these data do not preclude the possibility that reprogramming factor induction of iCMs may play an important role in myocardial regeneration and cardiac recovery from myocardial infarction, the ability of Gata4 to thereby inhibit postinfarct fibrosis may be an important new means of treating heart failure independently of the recent focus on the role of Gata4 as a cellular reprogramming factor. Additional observations that Gata4 also possesses primary cardiomyocyte-related inotropic and structural effects should be the subject of further investigations.

\section{Conflict of Interest Statement}

Authors have nothing to disclose with regard to commercial support.

The authors thank Shuyun Deng, MD, from the Gene Vector Core at Baylor College of Medicine, for the preparation of viral vectors, and Qianzi Zhang, MPH, for help with the statistical analysis.

\section{References}

1. Mathison M, Gersch RP, Nasser A, Lilo S, Korman M, Fourman M, et al. In vivo cardiac cellular reprogramming efficacy is enhanced by angiogenic preconditioning of the infarcted myocardium with vascular endothelial growth factor. J Am Heart Assoc. 2012;1:e005652.

2. Mathison M, Singh VP, Gersch RP, Ramirez MO, Cooney A, Kaminsky SM, et al. "Triplet" polycistronic vectors encoding Gata4, Mef2c, and Tbx5 enhances postinfarct ventricular functional improvement compared with singlet vectors. J Thorac Cardiovasc Surg. 2014;148:1656-64.e2.

3. Qian L, Huang Y, Spencer CI, Foley A, Vedantham V, Liu L, et al. In vivo reprogramming of murine cardiac fibroblasts into induced cardiomyocytes. Nature. 2012;485:593-8.

4. Song K, Nam YJ, Luo X, Qi X, Tan W, Huang GN, et al. Heart repair by reprogramming non-myocytes with cardiac transcription factors. Nature. 2012; 485:599-604.

5. Inagawa K, Miyamoto K, Yamakawa H, Muraoka N, Sadahiro T, Umei T, et al. Induction of cardiomyocyte-like cells in infarct hearts by gene transfer of Gata4, Mef2c, and Tbx5. Circ Res. 2012;111:1147-56.

6. Leask A, Abraham DJ. TGF- $\beta$ signaling and the fibrotic response. FASEB J. 2004; 18:816-27.

7. Crabos M, Roth M, Hahn AW, Erne P. Characterization of angiotensin II receptors in cultured adult rat cardiac fibroblasts. Coupling to signaling systems and gene expression. J Clin Invest. 1994;93:2372-8.

8. Merika M, Orkin SH. DNA-binding specificity of GATA family transcription factors. Mol Cell Biol. 1993;13:3999-4010.

9. Heineke J, Auger-Messier M, Xu J, Oka T, Sargent MA, York A, et al. Cardiomyocyte GATA4 functions as a stress-responsive regulator of angiogenesis in the murine heart. J Clin Invest. 2007;117:3198-210.

10. Rysä J, Tenhunen O, Serpi R, Soini Y, Nemer M, Leskinen H, et al. GATA-4 is an angiogenic survival factor of the infarct heart. Cir Heart Fail. 2010;3:440-50.
11. Nieto MA. The Snail superfamily of zinc-finger transcription factors. Nat Rev Mol Cell Biol. 2002;3:155-66.

12. Grande MT, Sánchez-Laorden B, López-Blau C, De Frutos CA, Boutet A, Arévalo M, et al. Snail-induced partial epithelial-to-mesenchymal transition drives renal fibrosis in mice and can be targeted to reverse established disease. Nat Med. 2015;21:989-97.

13. Olmeda D, Jordá M, Peinado H, Fabra Á, Cano A. Snail silencing effectively suppresses tumour growth and invasiveness. Oncogene. 2007;26:1862-74.

14. Stefanovic S, Christoffels VM. GATA-dependent transcriptional and epigenetic control of cardiac lineage specification and differentiation. Cell Mol Life Sci. 2015;72:3871-81.

15. Ieda M, Fu JD, Delgado-Olguin P, Vedantham V, Hayashi Y, Bruneau BG, et al. Direct reprogramming of fibroblasts into functional cardiomyocytes by defined factors. Cell. 2010;142:375-86.

16. Mori T, Kawara S, Shinozaki M, Hayashi N, Kakinuma T, Igarashi A, et al. Role and interaction of connective tissue growth factor with transforming growth factor- $\beta$ in persistent fibrosis: a mouse fibrosis model. J Cell Physiol. 1999; 181:153-9.

17. Hinz B, Phan SH, Thannickal VJ, Prunotto M, Desmoulière A, Varga J, et al. Recent developments in myofibroblast biology paradigms for connective tissue remodeling. Am J Pathol. 2012;180:1340-55.

18. Darby IA, Zakuan N, Billet F. Desmoulière. The myofibroblast, a key cell in normal and pathological tissue repair. Cell Mol Life Sci. 2016;73:1145-57.

19. Song G, Pacher M, Balakrishnan A, Yuan Q, Tsay HC, Yang D, et al. Direct reprogramming of hepatic myofibroblasts into hepatocytes in vivo attenuates liver fibrosis. Cell Stem Cell. 2016;18:797-808.

20. Oka T, Maillet M, Watt AJ, Schwartz RJ, Aronow BJ, Duncan SA, et al. Cardiac-specific deletion of Gata4 reveals its requirement for hypertrophy, compensation, and myocyte viability. Circ Res. 2006;98:837-45.

21. Zhou P, He A, Pu WT. Regulation of GATA4 transcriptional activity in cardiovascular development and disease. Curr Top Dev Biol. 2012;100:143-69.

22. He A, Gu F, Hu Y, Ma Q, Ye LY, Akiyama JA, et al. Dynamic GATA4 enhancers shape the chromatin landscape central to heart development and disease. Nat Commun. 2014;5:4907.

23. Stefanovic S, Barnett P, van Duijvenboden K, Weber D, Gessler M, Christoffels VM. GATA-dependent regulatory switches establish atrioventricular canal specificity during heart development. Nat Commun. 2014;5:3680.

24. Kuo CT, Morrisey EE, Anandappa R, Sigrist K, Lu MM, Parmacek MS, et al. GATA4 transcription factor is required for ventral morphogenesis and heart tube formation. Genes Dev. 1997;11:1048-60.

25. Trivedi CM, Zhu W, Wang Q, Jia C, Kee HJ, Li L, et al. Hopx and Hdac2 interact to modulate Gata4 acetylation and embryonic cardiac myocyte proliferation. Dev Cell. 2010;19:450-9.

26. Jayawardena TM, Finch EA, Zhang L, Zhang H, Hodgkinson CP, Pratt RE, et al. MicroRNA induced cardiac reprogramming in vivo: evidence for mature cardiac myocytes and improved cardiac function. Circ Res. 2015;116:418-24.

27. Bisping E, Ikeda S, Kong SW, Tarnavski O, Bodyak N, McMullen JR, et al. Gata4 is required for maintenance of postnatal cardiac function and protection from pressure overload-induced heart failure. Proc Natl Acad Sci U S A. 2006;103: 14471-6.

28. Bian J, Popović ZB, Benejam C, Kiedrowski M, Rodriguez LL, Penn MS. Effect of cell-based intercellular delivery of transcription factor GATA4 on ischemic cardiomyopathy. Circ Res. 2007;100:1626-33.

29. Lamouille S, Xu J, Derynck R. Molecular mechanisms of epithelialmesenchymal transition. Nat Rev Mol Cell Biol. 2014;15:178-96.

30. Peinado H, Ballestar E, Esteller M, Cano A. Snail mediates E-Cadherin repression by the recruitment of the Sin3A/histon deacetylase 1 (HDAC1)/ HDAC2 complex. Mol Cell Biol. 2004;24:306-19.

31. Singh VP, Mathison M, Patel V, Sanagasetti D, Gibson BW, Yang J, et al. MiR-590 promotes transdifferentiation of porcine and human fibroblasts toward a cardiomyocyte-like fate by directly repressing specificity protein 1. J Am Heart Assoc. 2016;5:e003922.

32. Mathison M, Singh VP, Chiuchiolo MJ, Sanagasetti D, Mao Y, Patel VB, et al. In situ reprogramming to transdifferentiate fibroblasts into cardiomyocytes using adenoviral vectors: implications for clinical myocardial regeneration. J Thorac Cardiovasc Surg. 2017;153:329-39.

Key Words: cardiac reprogramming, Gata4, fibrosis, Snail 


\section{Snail promoter}

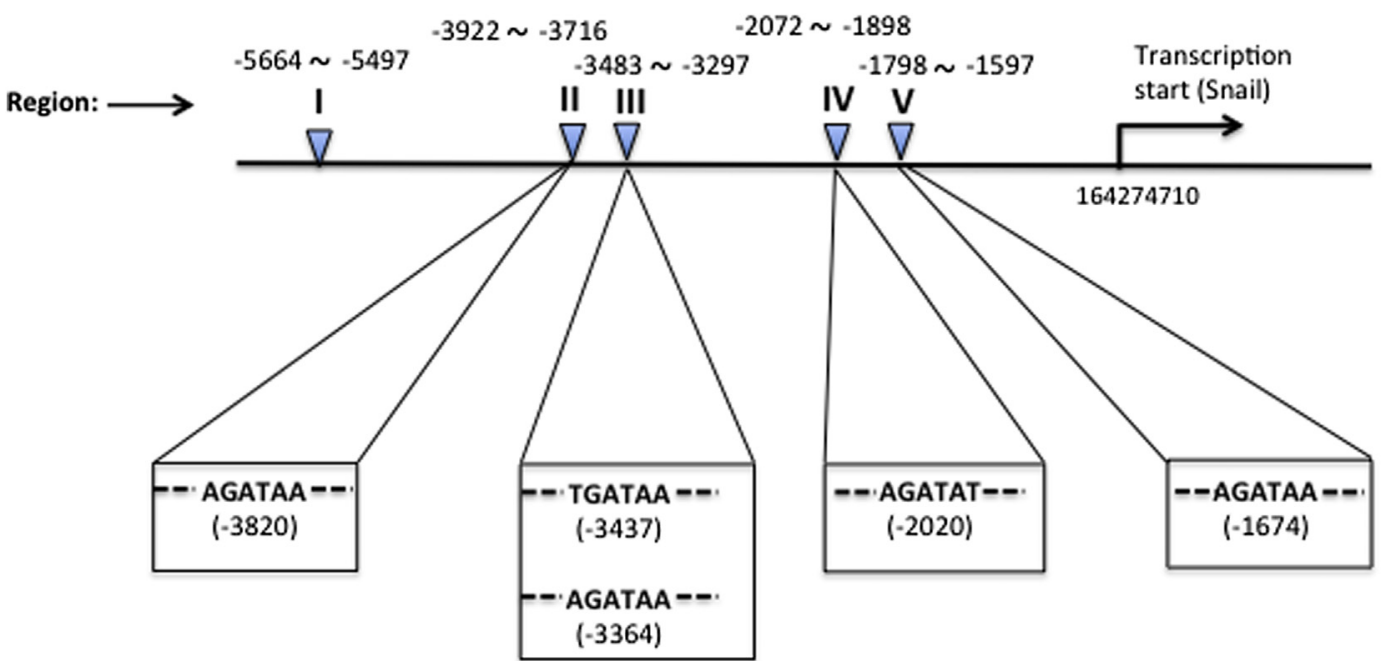

FIGURE E1. Schematic diagram of Snail promoter and Gata4 binding regions. Represented are locations of 4 theoretic Gata4 binding regions (regions II, III, IV, and V) and 1 region (region I) in which 5 sequences (AGATAA, TGATAA, AGATAA, AGATAT, and AGATAA) are not included. Further details of analysis are provided in the "Materials and Methods" section.



FIGURE E2. Distribution of baseline EFs. Graph depicts EF of each of 23 animal 3 days after coronary ligation and vector administration (4 animals died after initial surgery). Upper dot line: upper quartile boundary. Middle dot line: median. Lower dot line: lower quartile boundary. Baseline EFs of animals excluded from further analysis are shown in red. 
Gata4

GMT

GFP

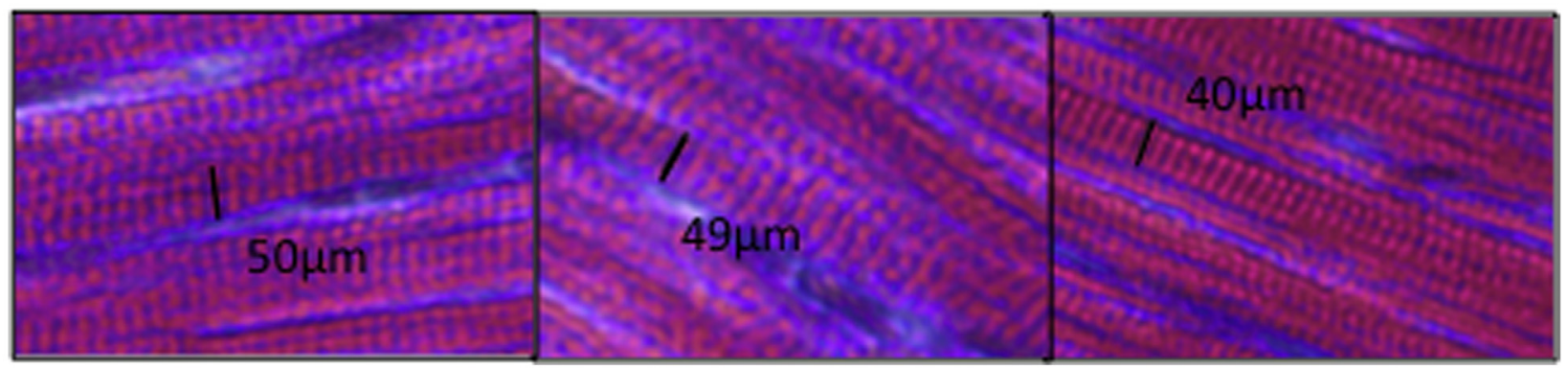

A

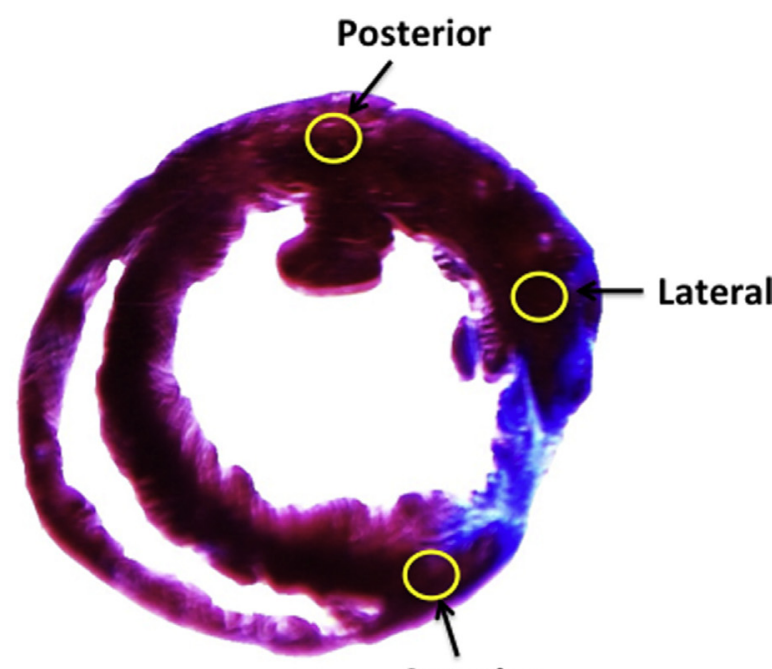

B

$$
\text { C }
$$



FIGURE E3. Cardiomyocyte diameter is greater in Gata4 and GMT treated versus control group. Cardiomyocyte diameter was measured from longitudinal images of cardiomyocytes, as described in the "Materials and Methods" section $(\times 400)$. A, Representative photomicrographs of cardiomyocytes after Gata4, GMT, or GFP control vector administration, as described in the "Materials and Methods" section. B, Counting was performed for 3 positions, anterior, lateral, and posterior, with 100 randomly chosen cardiomyocytes per each position. C, Graph depicts cardiomyocyte diameter quantification. $* P<.05$. **P $<.01, * * * P<.001$. GMT, Gata4, Mef2c, and Tbx5; GFP, green fluorescent protein. 


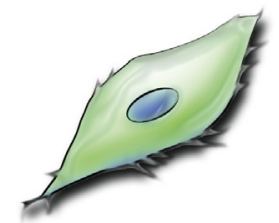

Resting fibroblast

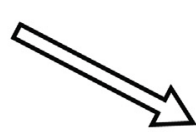

\section{Snail Upregulation}

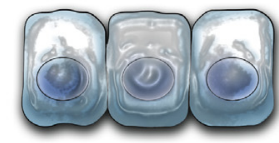

Epithelial cells
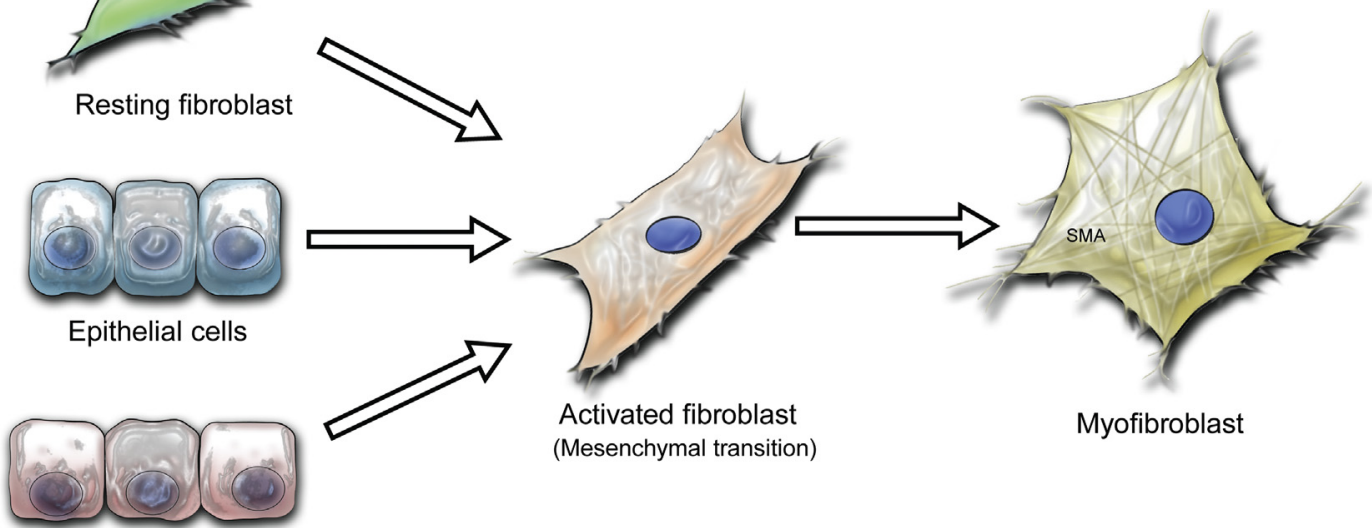

Endothelial cells

EMT-like process by Snail upregulation

AFMyT by persistent Snail

\section{A}

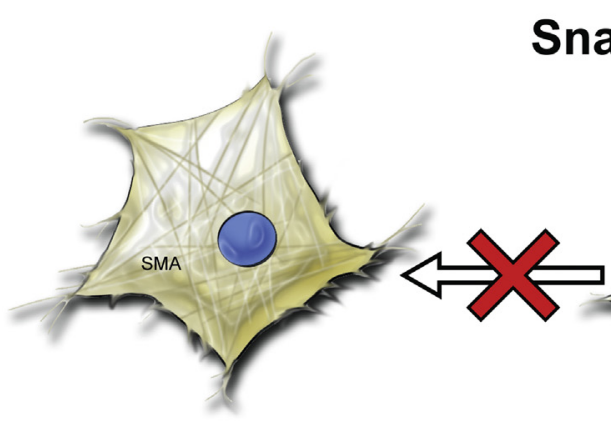

Myofibroblast
Snail downregulation


Activated fibroblas (Mesenchymal transition)

B AFMyT termination by Snail Downregulation
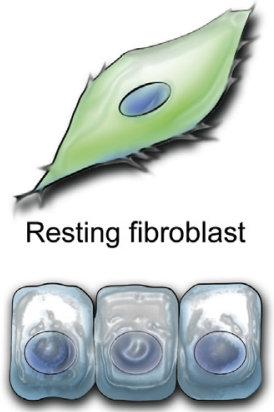

Epithelial cells

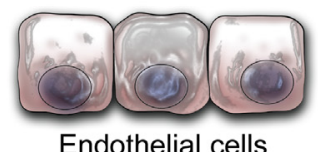

Endothelial cells

- MET by Snail downregulation -

FIGURE E4. A proposed mechanism of fibrosis as a result of EMT/MET-like transitions. Snail upregulation initiates loss of epithelial properties and acquisition of mesenchymal properties in epithelial cells (EMT), endothelial cells (endothelial-mesenchymal transition), or fibroblasts ("EMT-like" process). Activated mesenchymal cells are further differentiated into MFs via mesenchymal-MF transition by persistent Snail upregulation. Snail downregulation terminates mesenchymal-MF transition and induces (antifibrotic) MET transition. EMT, Epithelial-mesenchymal transition; $M E T$, mesenchymal-epithelial transition. 